\title{
CHANGES IN EMISSION LINE INTENSITIES OF HBV475
}

Shin'ichi Tamura

Astronomical Institute, Tohoku University, Sendai, JAPAN

Some of the results in the spectroscopic observation of HBV475 are presented.

Relative emission line intensities to $\mathrm{H}_{\beta}$ are obtained by quantitative analysis (Tamura, 1977, submitted to Astrophysical Letters). The most conspicuous character of these spectra are (i) appearance of emission lines of $\mathrm{Fe}^{+6}$ and $\mathrm{Ca}^{+4}$, (ii) strengthening of $\mathrm{HeII} \lambda 4685$ in com parison with Andrillat (1973, Mem.Soc.R.Sciences Liège, VI., 5, 371). I ( [FeVII] $\lambda 6087) / I([F e V I I] \lambda 5159)$ suggests that the electron density in the $\mathrm{Fe}^{+6}$ zone is $10^{6}$ to $10^{7} \mathrm{~cm}^{-3}$ if an electron temperature $\mathrm{T}_{\mathrm{e}}=20,000^{\circ} \mathrm{K}$ is assumed. The electron temperature of the $0^{+2}$ zone is about $6,000^{\circ} \mathrm{K}$ from the intensity ratio of $0^{+2}$ if we assume the electron density is greater than $10^{6} \mathrm{~cm}^{-3}$ from the absence of [OII] doublet. I $(\mathrm{N} 1+\mathrm{N} 2) / \mathrm{I}\left(\mathrm{H}_{\beta}\right)$ and $\mathrm{I}(\mathrm{HeII} \lambda 4685) / \mathrm{I}\left(\mathrm{H}_{\beta}\right)$ show different excitation classes, namely, 3 and 8 .

It may be considered that (a) the temperature of an exciting star of nebular matter is progressively increasing, (b) an ionization stratification is established by $\mathrm{Fe}^{+6}, \mathrm{He}^{+2}$, and $0^{+2}$. One can recognize the existence of mass motion from emission line profiles of [FeVII] in a relatively high dispersion spectrum (Tamura, in preparation).

In conclusion, HBV475 gives us a scene of planetary nebula formation since rapid change of the exciting star and mass ejection from it are suggested even now by spectral variations.

DENSITY DISTRIBUTION AND CHEMICAL ABUNDANCES IN NGC 7027

Andrea Preite-Martinez* and Nino Panagiat*

*Laboratorio di Astrofisica Spaziale, Frascati, Italy,

†Laboratorio di Radioastronomia, Bologna, Italy

The data available on permitted and forbidden line intensities of the planetary nebula NGC 7027 have been analyzed in terms of non-constant density models. The significance of the derived density distribution is discussed. Some implications to the determination of the chemical abundances are also discussed. 